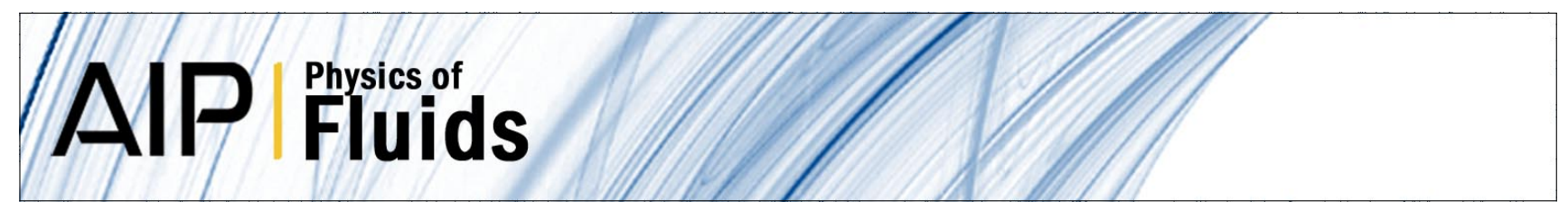

\title{
Ferrofluid droplet heating and vaporization under very large magnetic power: A thermal boundary layer model
}

C. F. C. Cristaldo and F. F. Fachini

Citation: Phys. Fluids 25, 037101 (2013); doi: 10.1063/1.4793611

View online: http://dx.doi.org/10.1063/1.4793611

View Table of Contents: http://pof.aip.org/resource/1/PHFLE6/v25/i3

Published by the AIP Publishing LLC.

\section{Additional information on Phys. Fluids}

Journal Homepage: http://pof.aip.org/

Journal Information: http://pof.aip.org/about/about_the_journal

Top downloads: http://pof.aip.org/features/most_downloaded

Information for Authors: http://pof.aip.org/authors

\section{ADVERTISEMENT}

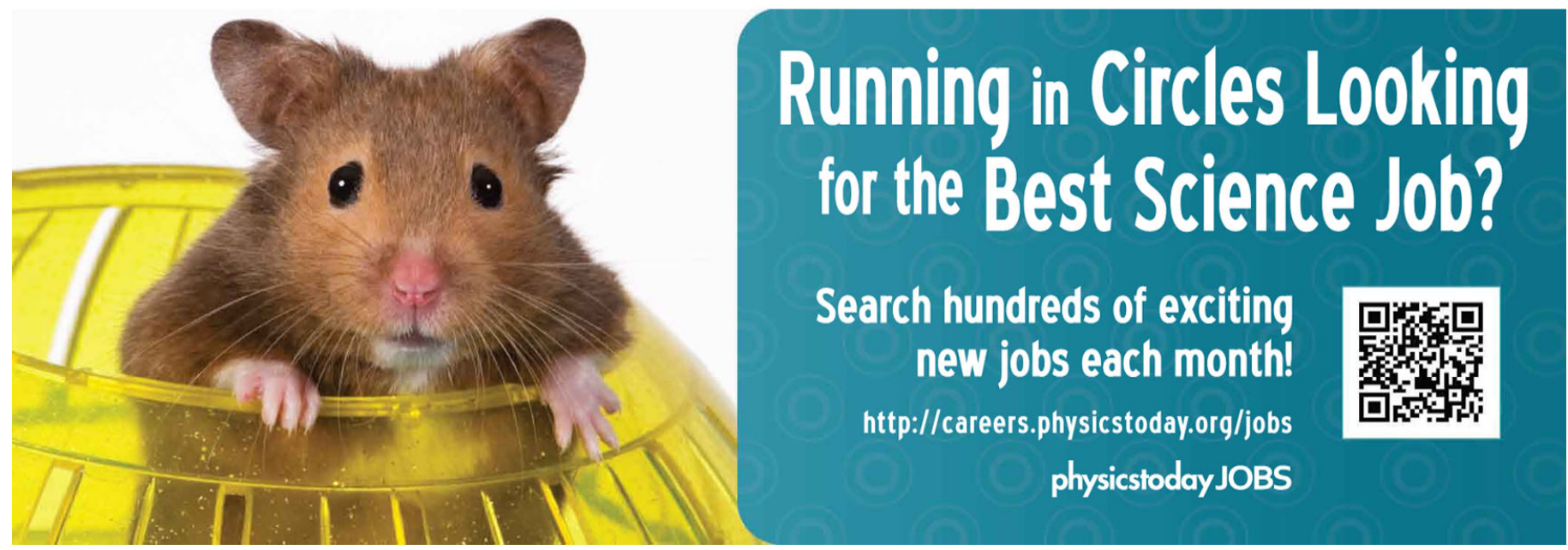




\title{
Ferrofluid droplet heating and vaporization under very large magnetic power: A thermal boundary layer model
}

\author{
C. F. C. Cristaldo a) and F. F. Fachinib) \\ Grupo de Mecânica de Fluidos Reativos, Laboratório de Combustão e Propulsão, \\ Instituto Nacional de Pesquisas Espaciais, 12630-000, Cachoeira Paulista, SP, Brasil
}

(Received 18 April 2012; accepted 13 February 2013; published online 4 March 2013)

\begin{abstract}
In this work, heating and vaporization of a liquid droplet with dispersed magnetic nanoparticles (ferrofluid) are analyzed. The ferrofluid droplet is in a quiescent inert gas phase with a temperature which is set down equal to, higher and lower than the liquid boiling temperature. Under these conditions, an alternating magnetic field is applied and, as a result, the magnetic nanoparticles generate heat by the Brownian relaxation mechanism. In this mechanism, the magnetic dipoles present a random orientation due to collisions between the fluid molecules and nanoparticles. The magnetic dipoles tend to align to the magnetic field causing rotation of the nanoparticles. Consequently the temperature increases due to the energy dissipated by the friction between the resting fluid and the rotating nanoparticles. Assuming a very large magnetic power and a uniform distribution of nanoparticles, the droplet core is uniformly heated. A thermal boundary layer is established in the liquid-phase adjacent to the droplet surface due to heat flux from the ambient atmosphere. The temperature profile inside the thermal boundary layer is obtained in appropriate time and length scales. In the present model, the ferrofluid droplet is heated up to its boiling temperature in a very short time. In addition, the combination of the heat generated by magnetic nanoparticles and heat conduction from gas phase results in a higher vaporization rate. Under specific conditions, the boiling temperature is achieved not at the surface but inside the thermal boundary layer. Moreover, the results point out that the thermal boundary layer depends directly on the vapor Lewis number but the vaporization rate reciprocally on it. @ 2013 American Institute of Physics. [http://dx.doi.org/10.1063/1.4793611]
\end{abstract}

\section{INTRODUCTION}

Fluids containing suspension of nanoparticles (nanofluids) have been extensively studied due to their enhanced physical properties, e.g., viscosity, specific heat, and thermal conductivity. ${ }^{1-3}$ Rapid augment in nanofluid applications has demanded the understanding of its properties and modelling. In the context of thermal problems, various theoretical models have been proposed for explaining the exceptional increase in the thermal conductivity. Empirical expressions for the effective thermal conductivity were proposed based on the thermal conductivities of the solid and liquid, their respective volume fractions and nanoparticle size.$^{4-6}$ More accurate models include effects of the nanoparticle-fluid interaction (nanolayer). ${ }^{7,8}$ The liquid molecules close to the nanoparticle surface form layered structures that behave much like a solid. One possible explanation for existence of the nanolayer is that the molecular structure of the liquid is more ordered in that region. Thus, a higher thermal local conductivity is expected in the liquid near the interface of the nanoparticle. ${ }^{9-11}$ Besides, nanofluids can present magnetic properties if magnetic nanoparticles are dispersed into the base liquid, known as magnetic nanofluids or ferrofluids. ${ }^{12-16}$

\footnotetext{
a) cristaldo@lcp.inpe.br.

b)fachini@lcp.inpe.br.
} 
The advantage of ferrofluids over non-magnetic nanofluids is the wide range of applications such as flow control, rupture into droplets, and heat transfer. For example, submitting ferrofluids to an external magnetic field gradient, the magnetic moments of the particles tend to align to the magnetic field and the ferrofluid is attracted to the region of the highest intensity field. Thus, ferrofluid flow can be driven by the mechanical motion of the magnetic field. An application for that is to pump a secondary fluid or to carry a body immersed in a ferrofluid. ${ }^{17-20}$ Another application is the rupture of the ferrofluid droplets into microdroplets by applying a uniform magnetic field. The magnetic field induces perturbations on the droplet surface, referred to as Rosenweig instability, that are responsible to break it up. In this application the magnetic field can be adjusted to control the final size of the micro-droplets. ${ }^{21}$ These ideas can also be applied to improve the spray atomization process. It is known, atomization is the key process in heating, vaporization and burn of liquid fuels, because the heat flux from gas phase to the droplets is directly dependent on the total spray liquid-gas interface area. $^{22-24}$

In the present model, the dispersion of the magnetic nanoparticles inside the droplet acts as a heat source (under the influence of an alternating magnetic field). This process is known as magneto relaxation heating. Magneto relaxation heating is the heating process produced by the energy dissipation due to friction between the rotating magnetic nanoparticles under the influence of an external magnetic field and the liquid surrounding them. From the droplet point of view, the interest is on the heating and vaporization imposed by both magnetic heating and heat transfer from gas phase. The conditions addressed in the problem consider a very larger magnetic power compared with the thermal power. As will be seen ahead, a thermal boundary layer is established in the liquid region adjacent to the droplet surface. It is worth mentioning that inside the thermal boundary layer, the magnetic and the thermal heat sources are of the same order, but in the droplet core the thermal power is negligible compared with the magnetic power.

Currently, magneto relaxation heating is used for inducing hyperthermia, which is used as an alternative treatment for destroying cancerous cells by increasing the temperature of tumor cells, known as magnetic hyperthermia. In this work, a new application for the magneto relaxation heating is proposed: the acceleration of the droplet heating process.

\section{PHYSICAL MODEL}

The magneto relaxation heating is based on the energy dissipated by magnetic nanoparticles when the ferrofluid is under the influence of an external alternating magnetic field. ${ }^{25,26}$ The energy dissipation of the nanoparticles is provided by two mechanisms: viscous dissipation due to rotation of the magnetic particle along with its magnetic dipole in a surrounding liquid (Brownian mechanism) and the augment of the nanoparticle temperature due to rotation of the magnetic dipole within the nanoparticle (Néel mechanism). The most important parameter that determines the magnetic nanoparticles heating rate is the effective relaxation time (the characteristic time of misalignment of the dipoles) defined as $t_{m}^{*-1}=t_{N}^{*-1}+t_{B}^{*-1}$, where $t_{N}^{*}$ and $t_{B}^{*}$ are the Néel and the Brownian relaxation times, respectively. Thus, the shorter relaxation time determines the dominating relaxation mechanism. In this analysis, the condition $t_{B}^{*} \ll t_{N}^{*}$ is assumed, hence the relaxation occurs mainly due to the Brownian mechanism.

The nanoparticles distribution inside the droplet is considered uniform in the present analysis. For a high magnetic heat source compared with that one provided by the heat flux from the gasphase, the temperature inside the droplet is uniform. ${ }^{27}$ In the region close to the droplet surface, both heat sources, magnetic heating and heat conduction from gas phase, have the same intensity. Consequently, a thermal boundary layer is established in that region during the droplet heating process. To follow the evolution of the thermal boundary layer, it is necessary to rescale the spatial and temporal coordinates. The solution for the thermal boundary layer matches the solution of the droplet core to that of the gas-phase, which is quasi-steady. The Brownian motion of nanoparticles and the regression of the droplet surface can cause agglomeration of nanoparticles on the droplet surface during the vaporization, ${ }^{28}$ but the agglomeration process is not taken into account in this analysis, as will be proved ahead. It is worth mentioning that the change in thermal conductivity 
due to the presence of nanoparticles inside the droplet is also neglected in this work with the aim of highlighting only the influence of the magnetic heating on the droplet heating.

\section{MATHEMATIC FORMULATION}

Whereas the ferrofluid droplet is in a quiescent ambient atmosphere, all processes in the liquid and gas phases present spherical symmetry and the problem can be considered as one-dimensional. The liquid phase presents the following properties: density $\rho_{l}$, specific heat $c_{l}$, and thermal conductivity $k_{l}$, which are considered constant. In a region far from the droplet surface, the following properties are constant: density $\rho_{\infty}^{*}$, temperature $T_{\infty}^{*}$, specific heat at constant pressure $c_{p}$, and thermal conductivity $k_{g_{\infty}}$. The liquid and gaseous phases are described by conservation equations, which are written in terms of the following nondimensional variables:

$$
\begin{gathered}
t \equiv \frac{t^{*}}{t_{c}^{*}}, \quad r \equiv \frac{r^{*}}{a^{*}(0)}, \quad \rho \equiv \frac{\rho^{*}}{\rho_{\infty}^{*}}, \\
\theta \equiv \frac{T^{*}}{T_{b}^{*}}, \quad u \equiv \frac{u^{*} a^{*}(0)}{\alpha_{\infty}}, \quad \text { and } \quad a \equiv \frac{a^{*}}{a^{*}(0)},
\end{gathered}
$$

in which $t, r, \rho, \theta, u$, and $a$ represent time, radial coordinate, density, temperature, gas velocity, and droplet radius, respectively. The superscript "*" stands for variables in dimensional form and the subscripts $b$ and $\infty$ stand for boiling and ambient conditions (far from the droplet), respectively. The time $t *$ is nondimensionalized by an estimated heating time $t_{c}^{*} \equiv\left[\left(a^{*}(0)\right)^{2} /\left(\alpha_{\infty} \epsilon\right)\right]$, with $\epsilon \equiv \rho_{\infty}^{*} / \rho_{l}^{*}$ and thermal diffusivity $\alpha_{\infty} \equiv k_{g_{\infty}} / c_{p} \rho_{\infty}^{*}$.

The conservation equations for mass and energy in the liquid phase are given by ${ }^{27}$

$$
\frac{d}{d t}\left(a^{3}\right)=-3 \lambda
$$

and

$$
\frac{\partial \theta}{\partial t}-\frac{A}{r^{2}} \frac{\partial}{\partial r}\left(r^{2} \frac{\partial \theta}{\partial r}\right)=P_{m} \frac{f^{2} t_{m}(\theta)}{1+\left(f t_{m}(\theta)\right)^{2}}
$$

in which $\lambda(t) \equiv \dot{m}\left(t^{*}\right) c_{p} /\left(4 \pi k_{g_{\infty}} a^{*}(0)\right)$ is the dimensionless vaporization rate, $\dot{m}\left(t^{*}\right)$ is the dimensional vaporization rate and $A \equiv c_{p} k_{l} / c_{l} k_{g_{\infty}}$. The right hand side of Eq. (2) represents the energy dissipation from magnetic nanoparticles under the influence of an alternating magnetic field. ${ }^{1}$ In the present model, the parameters that control the energy dissipation are the magnetic field frequency $f \equiv 2 \pi f^{*} t_{B_{b}}^{*}$, the effective relaxation time $t_{m} \equiv t_{m}^{*} / t_{B_{b}}^{*}$ and the ratio of the magnetic heat source to the thermal source $P_{m}$. The frequency $f^{*}$ is nondimensionalized by effective relaxation time determined at the boiling temperature $t_{B_{b}}^{*}$. The magnetic parameter $P_{m}$ is defined as ${ }^{27}$

$$
P_{m} \equiv \frac{\mu_{0} \chi_{0} H_{0}^{2} / 2}{\rho_{l} c_{l} T_{b}^{*}} \frac{t_{c}^{*}}{t_{B_{b}}^{*}}
$$

in which $\mu_{0}$ is the magnetic permeability $\left(\mu_{0}=4 \pi \times 10^{-7} \mathrm{Tm} / \mathrm{A}\right), H_{0}$ is the magnetic field amplitude, and the magnetic susceptibility $\chi_{0}$ is described by Langevin equation

$$
\chi_{0}=\chi_{i} \frac{3}{\xi}\left(\operatorname{coth} \xi-\frac{1}{\xi}\right) .
$$

The initial susceptibility is given by $\chi_{i} \equiv \mu_{0} \phi M_{d}^{2} V_{N} /\left(3 \kappa T^{*}\right)$, in which $\phi, M_{d}, V_{N}$, and $\kappa$ are volume fraction of nanoparticles, domain magnetization, nanoparticles volume, and Boltzmann constant $\left(1.38 \times 10^{-23} \mathrm{~J} / \mathrm{K}\right)$, respectively. The Langevin parameter $\xi$ is defined as $\xi=\mu_{0} M_{d} H V_{N} /\left(\kappa T^{*}\right)$ with $H=H_{0} \cos \left(2 \pi f^{*} t *\right)$. The equilibrium susceptibility $\chi_{0}$ is a conservative estimative for low limit of the source term in Eq. (2). ${ }^{1}$ Therefore, $\chi_{0}$ can be assumed to be a constant. This assumption is valid because the volume fraction of nanoparticles is constant during the heating period, as will be seen ahead. The Brownian relaxation time is related to the inverse of temperature and is defined as $t_{B}^{*} \equiv 3 \eta V_{H} / \kappa T^{*}$, where $\eta$ is the viscosity of the surrounding liquid, and $V_{H}$ is the hydrodynamic 

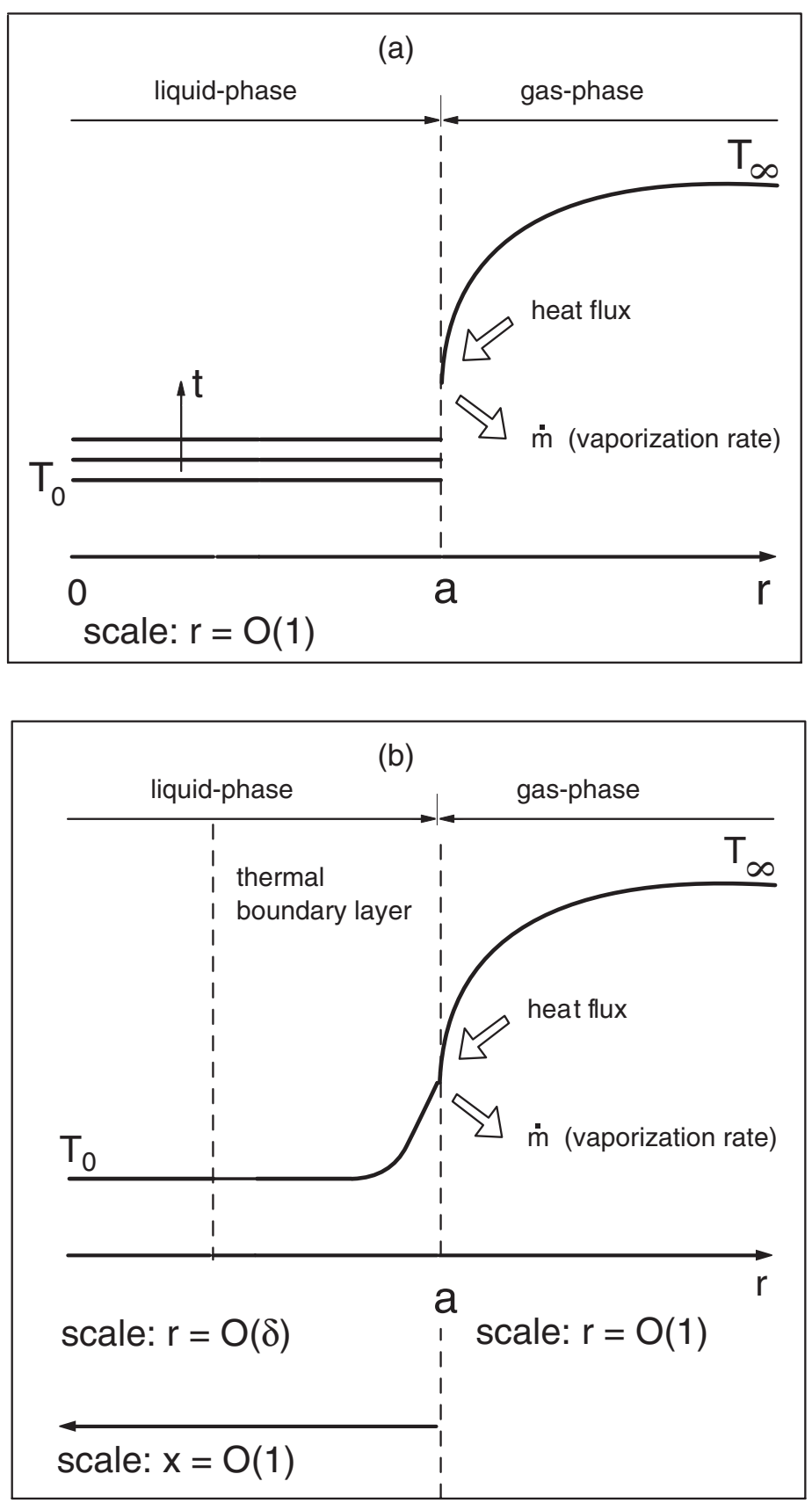

FIG. 1. Schematic representation of the temperature profile of the problem: (a) Liquid and gas phases in spatial coordinate $r$. (b) Liquid phase in spatial coordinate $x$ and gas phase in spatial coordinate $r$.

volume of the particle. The hydrodynamic volume is the effective volume that includes the volume of the coating layer of the nanoparticle. The Brownian relaxation time $t_{B}^{*}$ is nondimensionalized by its value at the boiling temperature leading to $t_{B} \equiv t_{B}^{*} / t_{B_{b}}^{*}=1 / \theta{ }^{27}$

The hypothesis that the magnetic power is much larger than the thermal power, $P_{m} \gg 1$, is assumed in this work. This assumption is valid for a field intensity of about $10^{-2}$ tesla. Under this condition, the temperature profile is uniform in the droplet core, varying just with time (Fig. 1(a)). ${ }^{27}$ However, in a thin zone adjacent to the droplet surface, the uniform behavior from the temperature profile of the droplet core changes to a time-spatial variation to match the temperature gradient 
imposed by the heat flux from the gas-phase (Fig. 1(b)). Consequently, a thermal boundary layer must be formed adjacent to the droplet surface. Therefore it is necessary to rescale not only the time but also the radial coordinate in Eqs. (1) and (2) to follow properly the evolution of the thermal boundary layer.

By analyzing Eq. (2), the appropriate time scale is $t \sim P_{m}^{-1}$, then the new time scale can be written as $\tau \equiv t P_{m}$, for $\tau=O(1)$. In addition, in order to describe the spatial variation of the temperature profile in the liquid phase, the following change of the spatial variable $r=a+\delta x$ is necessary. As will be shown, the thickness $\delta$ is very small, $\delta \sim P_{m}^{-1 / 2} \ll 1$. According to classical procedure, a thermal boundary layer is established in the thickness $\delta$, which is described by the variable $x=O(1)$, as exhibited in the schematic Fig. 1(b).

Equations (1) and (2) in these new variables are written as

$$
\frac{d}{d \tau}\left(a^{3}\right)=-3 \frac{\lambda}{P_{m}}
$$

and

$$
\frac{\partial \theta}{\partial \tau}+\frac{1}{\delta} \frac{d a}{d \tau} \frac{\partial \theta}{\partial x}-\frac{A}{\delta^{2} P_{m}} \frac{\partial^{2} \theta}{\partial x^{2}}=\frac{f^{2} \theta}{\theta^{2}+f^{2}} .
$$

By analyzing asymptotically the source term $S(\theta ; f) \equiv f^{2} \theta /\left(f^{2}+\theta^{2}\right)$, it is observed $S(\theta ; f) \sim f^{2} / \theta$ $\ll 1$ and $S(\theta ; f) \sim \theta$ for low $(f \ll 1)$ and high $(f \gg 1)$ frequencies, respectively. For $\delta=\left(A / P_{m}\right)^{1 / 2}$, all terms in Eq. (6) become order unity, except the convective term that presents the order $\left(A P_{m}\right)^{-1 / 2}$ $\ll 1$ because $d a / d \tau \sim P_{m}^{-1}$ for $a \sim 1$. Therefore the effect of the convection due to the variation of the radius is negligible whereas $a \gg P_{m}^{-1 / 2}$ according to Eq. (5).

Thus, by imposing $\delta=\left(A / P_{m}\right)^{1 / 2}$, Eq. (6) takes the form

$$
\frac{\partial \theta}{\partial \tau}-\frac{\partial^{2} \theta}{\partial x^{2}}=\frac{f^{2} \theta}{\theta^{2}+f^{2}} .
$$

This equation and the following boundary conditions:

$$
\frac{\partial \theta}{\partial x}=0 \quad \text { for } \quad x \rightarrow-\infty
$$

and

$$
\left.a^{2} \theta^{n} \frac{\partial \theta}{\partial r}\right|_{a^{+}}=\left.\left(A P_{m}\right)^{1 / 2} a^{2} \frac{\partial \theta}{\partial x}\right|_{x=0^{-}}+\lambda L \quad \text { at } \quad r=a
$$

describe the evolution of the temperature profile inside the thermal boundary layer. In this analysis, the gas thermal conductivity is considered to be a function of temperature, according to $k_{g} / k_{g_{\infty}}=\theta^{n}$ with $n=0.5$. The dimensionless latent heat of vaporization is defined as $L \equiv L^{*} /\left(c_{p} T_{b}\right)$, where $L^{*}$ is the latent heat of vaporization. Equation (9) represents the energy conservation at the droplet surface: part of the heat that is supplied by the ambient atmosphere to the droplet is used for the droplet heating and part for the vaporization process.

Based on the gas phase radial flow from the droplet to the ambient atmosphere, the gas-phase conservation equations are presented below: ${ }^{29,30}$

$$
\begin{gathered}
\epsilon P_{m} \frac{\partial \rho}{\partial \tau}+\frac{1}{r^{2}} \frac{\partial}{\partial r}\left(r^{2} \rho u\right)=0 \\
\epsilon P_{m} \rho \frac{\partial Y}{\partial \tau}+\rho u \frac{\partial Y}{\partial r}-\frac{1}{r^{2}} \frac{\partial}{\partial r}\left(\frac{r^{2} \theta^{n}}{L e} \frac{\partial Y}{\partial r}\right)=0 \\
\epsilon P_{m} \rho \frac{\partial \theta}{\partial \tau}+\rho u \frac{\partial \theta}{\partial r}-\frac{1}{r^{2}} \frac{\partial}{\partial r}\left(r^{2} \theta^{n} \frac{\partial \theta}{\partial r}\right)=0
\end{gathered}
$$

in which $Y$ is the vapor mass fraction and $L e$ is the vapor Lewis number. In this work, under low pressure conditions, $\epsilon \equiv \rho_{\infty} / \rho_{l}$ is considered small enough, such that the condition $\epsilon P_{m} \ll 1$ is still 
found. Hence, the gas phase processes can be treated as quasi-steady-state. Thus the conservation equations can be rewritten as

$$
\begin{gathered}
r^{2} \rho u=\lambda \\
\frac{\lambda}{r^{2}} \frac{\partial Y}{\partial r}-\frac{1}{r^{2}} \frac{\partial}{\partial r}\left(\frac{r^{2} \theta^{n}}{L e} \frac{\partial Y}{\partial r}\right)=0, \\
\frac{\lambda}{r^{2}} \frac{\partial \theta}{\partial r}-\frac{1}{r^{2}} \frac{\partial}{\partial r}\left(r^{2} \theta^{n} \frac{\partial \theta}{\partial r}\right)=0 .
\end{gathered}
$$

The boundary conditions at the surface are described by Eq. (9) and

$$
-\frac{r^{2} \theta^{n}}{L e} \frac{\partial Y}{\partial r}=\lambda\left(1-Y_{s}\right), \quad \text { at } \quad r=a^{+} .
$$

The superscript + stands for the condition at the surface in the gas-side and $Y_{S}$ is the vapor mass fraction at the droplet surface. In the region far from the droplet, the following boundary conditions hold

$$
\theta=\theta_{\infty}, \quad \text { and } \quad Y=0 \quad \text { as } \quad r \rightarrow \infty
$$

At the liquid-gas interface, vapor and liquid are assumed to be in equilibrium. For this reason, the Clausius-Clapeyron relation

$$
Y_{s}=\exp \left[\gamma\left(1-\theta_{b} / \theta_{s}\right]\right.
$$

can be used to relate the vapor mass fraction at the surface to the surface temperature $\theta_{s}$, where $\gamma \equiv L^{*} M_{w} /\left(R_{g} T_{b}^{*}\right)$ depends on the liquid molecular weight $M_{w}$ and universal gas constant $R_{g}$. Equations (14) and (15) can be integrated once considering the boundary conditions given by Eqs. (16) and (17) and the energy conservation at the interface, Eq. (9), resulting in the following system

$$
\begin{gathered}
\frac{\partial \theta}{\partial r}=\frac{\lambda\left(\theta-\theta_{s}+L\right)+Q^{-}}{r^{2} \theta^{n}}, \\
\frac{\partial Y}{\partial r}=-\frac{\lambda(1-Y) L e}{r^{2} \theta^{n}}
\end{gathered}
$$

in which $Q^{-} \equiv\left(A P_{m}\right)^{1 / 2} a^{2}(\partial \theta / \partial x)_{x=0^{-}}$is the heat flux from surface to inner region of the droplet.

\section{NUMERICAL STRATEGY}

A ferrofluid droplet with initial radius $a_{0}=1$ is in a quiescent inert atmosphere at temperature $\theta_{\infty}$ and vapor mass fraction $Y_{\infty}$. An alternating magnetic field is applied with frequency $f$. In this problem, the term "ambient temperature" means the temperature of a region far from the droplet. It is worth mentioning that the spatial scale of the liquid phase inside the thermal boundary layer is $r$ $=O(\delta)$ or $x=O(1)$, and the gas phase is solved in the scale $r=O(1)$. For the numerical solution of the problem the following procedure is employed:

(1) First, the energy conservation equation for the liquid phase, Eq. (7), is integrated with arbitrary values for the surface temperature $\theta_{s}$ and vaporization rate $\lambda$ and, as a result, $Q^{-}$is calculated.

(2) Using these values of $\theta_{s}, \lambda$ and $Q^{-}$, the integration of Eqs. (19) and (20) is performed from the droplet surface $r=a$ to a region far from the droplet $r \gg 1$.

(3) If the boundary conditions $\theta_{\infty}$ and $Y_{\infty}$ are not satisfied, new estimates for $\theta_{s}$ and $\lambda$ are obtained by the Newton-Raphson procedure and the process is repeated until the boundary conditions are satisfied.

(4) At this point, the process is advanced to the next time step. 
Besides that, at each time step, the droplet radius is calculated using Eq. (5). According to this equation, the radius has a very small variation in $\tau=O(1)$. Even for $P_{m} \gg 1$, the vaporization rate $\lambda$ is of the order unity because the heat flux from gas phase is order unity, as shown in Eq. (9). Therefore the heating process occurs practically with no expressive variation of the droplet radius. This justifies the volume fraction of magnetic nanoparticles to be considered constant during the heating process.

\section{RESULTS AND DISCUSSION}

The base fluid used in this work is n-heptane at initial temperature of $297 \mathrm{~K}$ or $\theta=0.8$. The constants properties of the $\mathrm{n}$-heptane are: density $\rho_{l}=6.8 \times 10^{5} \mathrm{~g} / \mathrm{m}^{3}$, thermal conductivity $k_{l}=0.609 \mathrm{~J} / \mathrm{m} \mathrm{s} \mathrm{K}$, specific heat $c_{l}=2.216 \mathrm{~J} / \mathrm{g} \mathrm{K}$, latent heat of vaporization $316.76 \mathrm{~J} / \mathrm{g}$, boiling temperature $T_{b}^{*}=371.15 \mathrm{~K}$. The magnetic nanoparticle is maghemite $\left(\gamma \mathrm{Fe}_{2} \mathrm{O}_{3}\right)$ of radius of $r_{N}$ $=10 \mathrm{~nm}$, thickness of coating layer $\delta=1 \mathrm{~nm}$, domain magnetization of $M_{d}=414 \mathrm{kA} / \mathrm{m}$ and volume fraction of nanoparticles $\phi=0.07$. The intensity of magnetic field $B_{0}=\mu_{0} H_{0}=1$ tesla. The model demands $P_{m} \gg 1$, then the results presented ahead are for droplet radius larger than $70 \mu \mathrm{m}$. For these properties the parameter $P_{m}$ is 100 . However, for $a<70 \mu \mathrm{m}, P_{m}$ decreases according to $P_{m} / 100 \sim(a / 70)^{2}$ with droplet radius. For example, for $a=10 \mu \mathrm{m}, P_{m}$ is about 2 , such condition demands the integration of Eq. (2) to describe the temperature evolution, the boundary layer approximation is no longer valid.

The heating process is followed until any part of the droplet reaches the boiling temperature $\theta=1$. This model does not allow the temperature in the droplet to be higher than the boiling temperature because any disturbance can generate bubbles in its interior and break it up. For all cases, the vapor mass fraction in the region far from the droplet is $Y_{\infty}=0$ and the Lewis number Le is equal to unity, except when another value is explicitly mentioned.

It is important to highlight that, under certain conditions, the magnetic source may heat up the droplet core and/or the thermal boundary layer faster than the droplet surface is heated by the surrounding gas. Then, a heat flux from the droplet interior to the droplet surface must be observed. The consequences are higher vaporization rates as well as a local maximum for the temperature profile located inside the thermal boundary layer. Hence, the boiling condition is achieved not at the droplet surface but inside the thermal boundary layer. The numerical simulation is ended at the time that the boiling temperature $(\theta=1)$ is achieved in any position of the droplet.

Figure 2(a) shows the heating time of the droplet as a function of the magnetic field frequency $f$ for different values of ambient temperature $\theta_{\infty}$. The frequency $f$ varies from $f=0.3$ to values for which the relaxation process of nanoparticles reaches the saturation state, $f \approx 5$. It is worth mentioning that, for $f<0.3$, the model of the thermal boundary layer becomes unrealistic because its thickness becomes close to one order of magnitude larger than $\left(A / P_{m}\right)^{1 / 2}$, precisely $\delta=\left(A / P_{m}\right)^{1 / 2} / f$, as pointed by Eq. (6). Since the magnetic source $S(\theta, f)$ increases with $f$, an increase of the magnetic field frequency reduces the time for the droplet to reach its boiling temperature. The pronounced influence of the ambient temperature on the heating time for low frequencies $(f<1.5)$ is observed. For high frequencies, however, the ambient temperature has litle influence on the heating time, as seen in Fig. 2(a).

Figure 2(b) shows the vaporization rate $\lambda_{b}$ as a function of the magnetic field frequency. The subscript $b$ from now on will stand for the variable value when any part of the droplet reaches the boiling condition. Unlike in the droplet heating time, the frequency of the magnetic field does not have an expressive influence on the vaporization rate, as seen in Fig. 2(b). Thus the vaporization rate is strongly dependent on the ambient temperature. Therefore, even for very intense magnetic heating, the vaporization rate keeps depending on the heat flux from the gas-phase, which explains the strong dependence on the ambient temperature.

Figure 2(c) shows the temperature at the droplet surface $\theta_{s b}$ as a function of the magnetic field frequency $f$. Like the vaporization rate, the droplet surface temperature is strongly dependent on the ambient temperature. For the condition of low ambient temperature and low frequencies $(f<1.5)$, as shown in Fig. 2(c) for $\theta_{\infty}=0.9$, the magnetic field frequency also plays an important role in determining $\theta_{s b}$. 

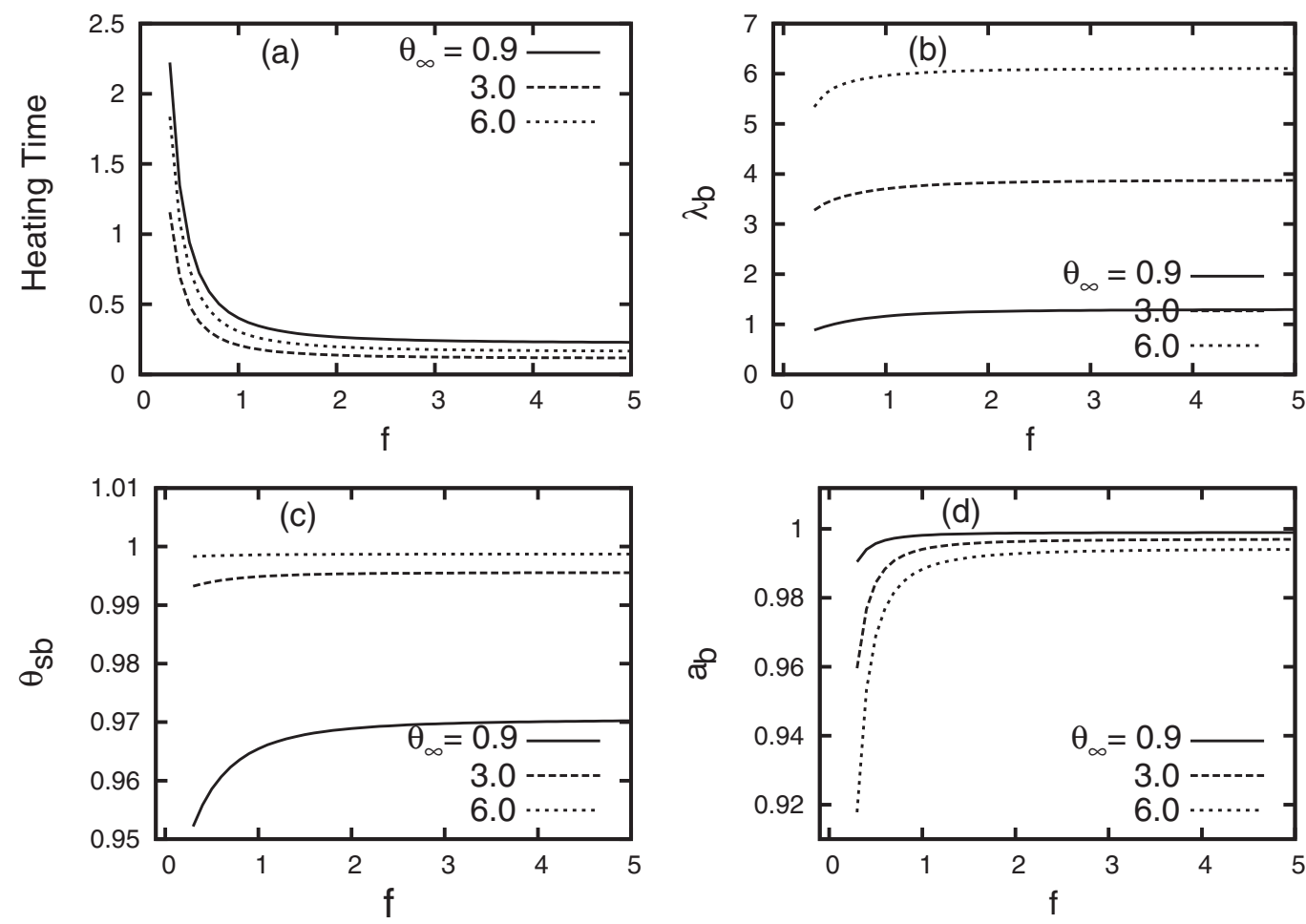

FIG. 2. Dependence of the droplet properties on the frequency of the magnetic field at the time in which any part of the droplet reaches the boiling temperature: (a) Heating time, (b) vaporization rate, (c) droplet surface temperature, and (d) droplet radius, for three different ambient temperatures.

Since the vaporization rate as well as the droplet surface temperature are controlled by the heat conservation at a very thin zone around the liquid-gas interface (droplet surface), the volumetric heat generation is negligible because of its dependence on the volume. This feature explain the strong dependence of the heating time with magnetic heating via the frequency and of the vaporization rate and droplet surface temperature with the heat flux from the gas-phase via the ambient temperature.

Figure 2(d) shows the droplet radius $a_{b}$ as a function of the magnetic field frequency using the same conditions as in the three previous plots. Since the heating time $(\tau=O(1))$ is very short, as seen in Fig. 2(a), the variation of the droplet radius during the heating time is very small. According to Eq. (5), it follows the relation $a_{b}^{3} \sim 1-3 \lambda / P_{m}$, a trend confirmed by Fig. 2(d). For low frequencies, the ambient temperature is responsible, through the ambient heat flux, for the increase of the vaporization rate and consequently the reduction of the droplet radius, hence $a_{b}$ is smaller, the larger the ambient temperature $\theta_{\infty}$ is. From Fig. 2, it is concluded that the magneto relaxation heating has a strong influence on the heating time but weak on the vaporization rate.

Next, the droplet heating by magnetic heating process is analyzed at different ambient atmosphere conditions, temperature and vapor mass fraction.

\section{A. High ambient atmosphere temperature}

This section analyzes heating and vaporization of droplet in an ambient atmosphere of $\theta_{\infty}=6$ and $Y_{F_{\infty}}=0$. These values describe the ambient atmosphere with high temperature, no oxidant and low fuel mass fraction found by droplets close to flames, due to the spray combustion. The effects of the magnetic source are quantified in the cases $f=0.3,1.0$, and 5.0. As can be seen in Fig. 3, the temperature inside the droplet is higher than that at the droplet surface. Therefore the temperature reaches the boiling condition inside the thermal boundary layer. This happens because, besides 

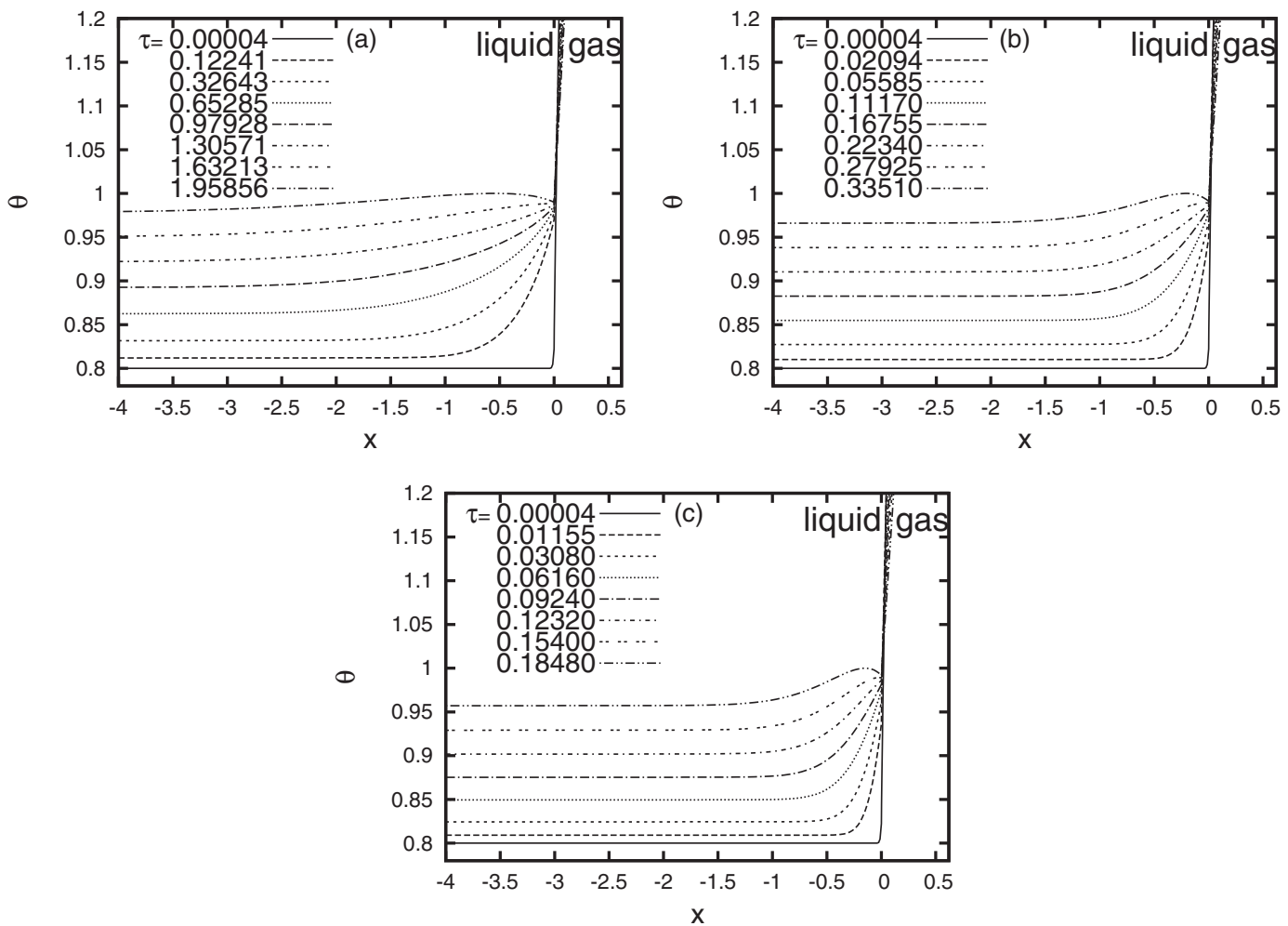

FIG. 3. Evolution of the temperature profile for three different magnetic field frequencies $f=0.3$ (a), 1.0 (b), and 5.0 (c) for $\theta_{\infty}=6.0$.

the magnetic heat source, the ambient heat flux is large enough to sustain high temperature in the thermal boundary layer even with the vaporization, which is responsible to decrease the temperature through mass loss. Under this ambient condition, the magnetic source $S(\theta, f)$, that depends on the local temperature, is able to increase the temperature of the thermal boundary layer for values higher than that of the droplet surface. This increase of temperature in adjacent layers in the liquid side of the droplet surface is also evidenced in a previous work, where a semi-transparent droplet is internally heated by absorption of radiation from high temperature ambient atmosphere. ${ }^{31}$ In the present analysis, in addition to the gas phase heat flux, heat flux from the thermal boundary layer $\left(Q^{-}\right)$to the droplet surface raises the vaporization rates of ferrofluid droplet to above that described by classical models. In such models $Q^{-}$is always positive but in the present work $Q^{-}$can be negative too.

The droplet surface temperature $\theta_{s}$ and the vaporization rate $\lambda$ as functions of time are shown in Figs. 4 and 5. It is verified that both properties rise rapidly for high frequency conditions.

For low frequency conditions, the vaporization rate seems to approach a low variation regime, as shown in Fig. 5. For $f=1.0$ and 5.0, the vaporization rate and the temperature at the droplet surface increases almost at the same rate (Fig. 4). This feature is a consequence of the extra heat flux from the thermal boundary layer to the droplet surface.

\section{B. Ambient atmosphere at boiling temperature}

The following results show the ferrofluid droplet in a gaseous environment at the boiling temperature, $\theta_{\infty}=1$. Temperature profiles in the thermal boundary layer are shown in Fig. 6 for magnetic field frequencies $f=0.3,1$, and 5. Since in these cases the heat flux from the ambient atmosphere is low, the magnetic heat source controls almost integrally the heating process. In addition, because the magnetic nanoparticles distribution is uniform, the droplet has practically 


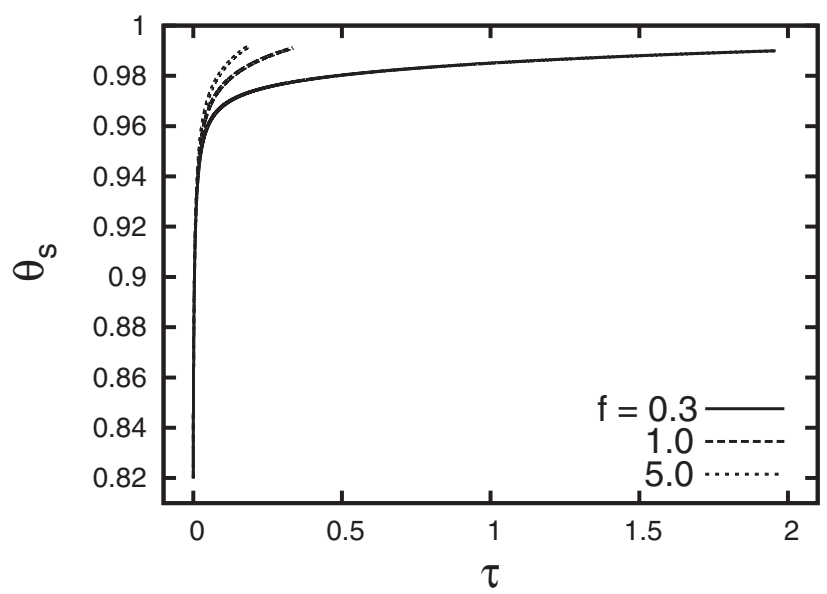

FIG. 4. Droplet surface temperature as a function of time for three different magnetic field frequencies $f=0.3$ (a), 1.0 (b), and 5.0 (c) for $\theta_{\infty}=6.0$.

a uniform heating, resulting in a thermal boundary layer that presents only a small temperature variation. The droplet surface temperature presented in Fig. 7 follows a similar behavior described by the analytical solution, ${ }^{27}$ except for low magnetic field frequency but at the end of the heating period. This indicates that the analytical solution is suitable for solving the problem in low ambient temperature condition.

Figure 8 shows the vaporization rate as a function of time. The results show a strong dependence of the vaporization rate on the magnetic field frequency. Unlike the surface temperature, the simulated and analytical values for the vaporization cannot be compared directly. The comparison will be done indirectly through the fuel mass fraction at the droplet surface. Figure 9 shows the excellent concordance between the simulated and calculated values.

\section{Low ambient atmosphere temperature}

The droplet heating in an ambient atmosphere with a lower temperature than the initial droplet temperature is analyzed, specifically the case $\theta_{\infty}=0.75$ is considered. The temperature profiles are shown in Fig. 10 for different values of magnetic field frequency.

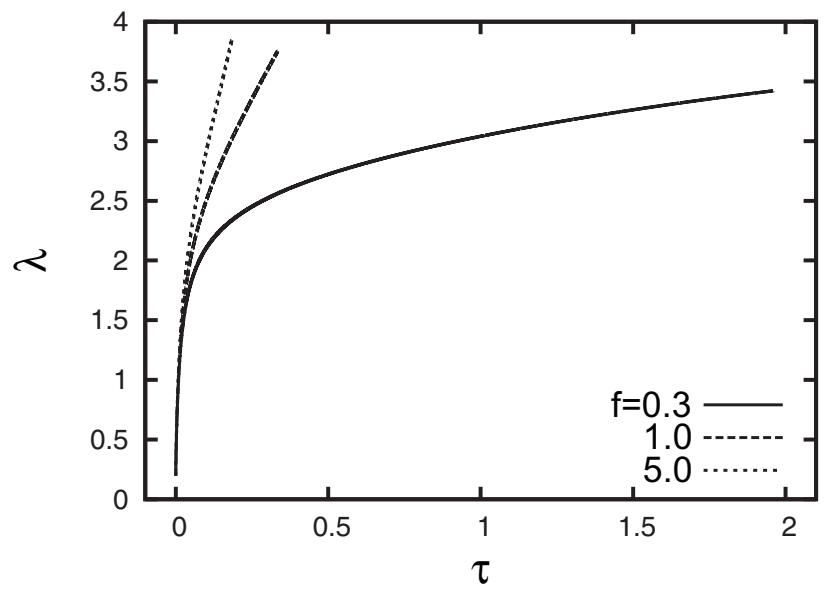

FIG. 5. Vaporization rate as a function of time for three different magnetic field frequencies $f=0.3$ (a), 1.0 (b), and 5.0 (c) for $\theta_{\infty}=6.0$. 

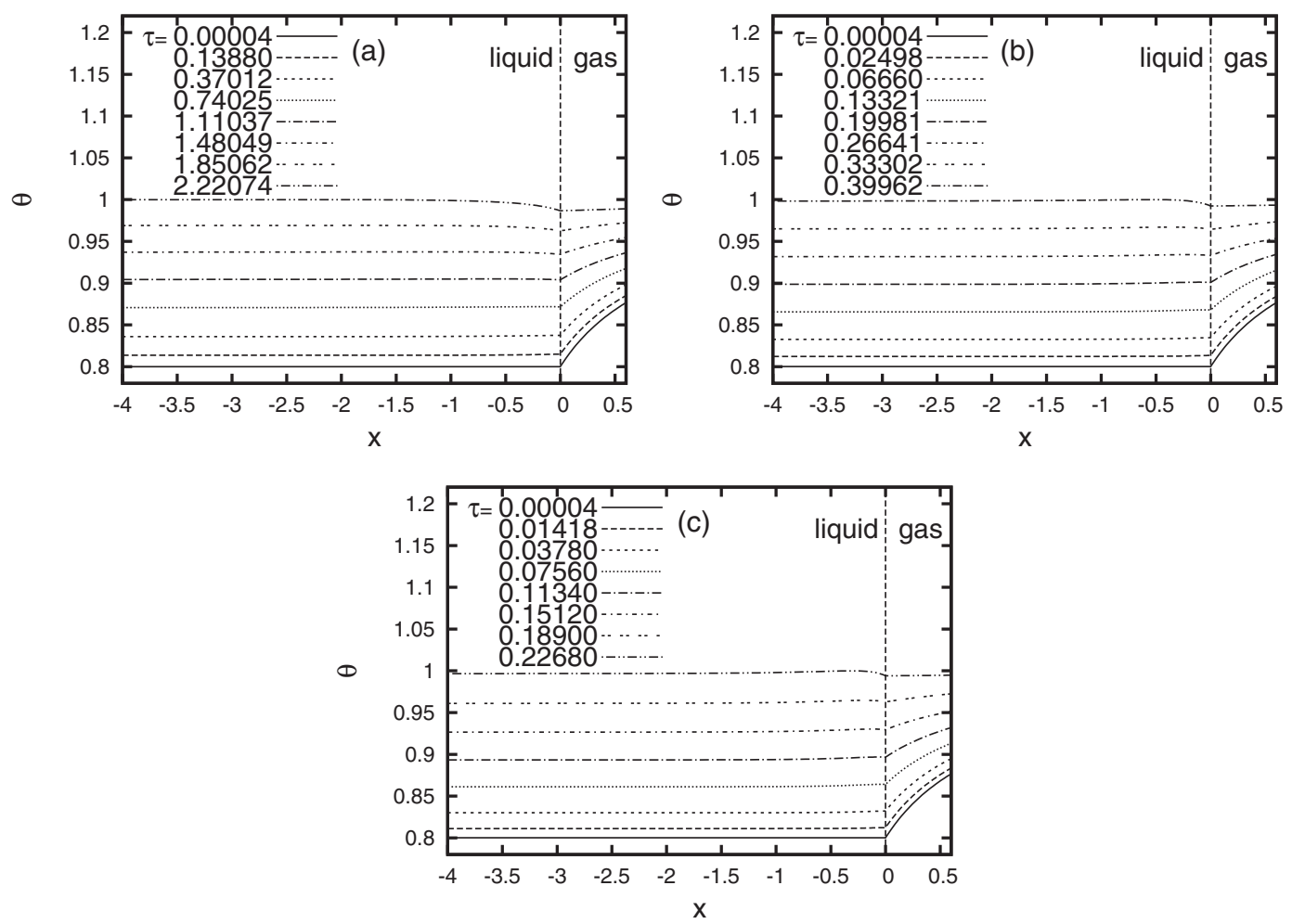

FIG. 6. Evolution of the temperature profile for three different magnetic field frequencies $f=0.3$ (a), 1.0 (b), and 5.0 (c) for $\theta_{\infty}=1.0$.

The results are similar to the previous case $\left(\theta_{\infty}=1\right)$, but the thermal boundary layer cannot be observed easily. Furthermore the heating time is practically the same as that for the ambient temperature $\theta_{\infty}=1$, as shown in Fig. 6. As this happens at low ambient temperature as well as $\theta_{\infty}$ $=1$, the droplet heating depends practically on the magnetic source. Hence, the heating time can be controlled just by the magnetic field frequency.

The small influence of the ambient temperature on the heating process can be quantified evaluating the heat flux across the droplet surface, which is given by $q^{-}=Q^{-} /\left(A P_{m}\right)^{1 / 2}=a^{2}(\partial \theta / \partial x)_{x=a^{-}}$.

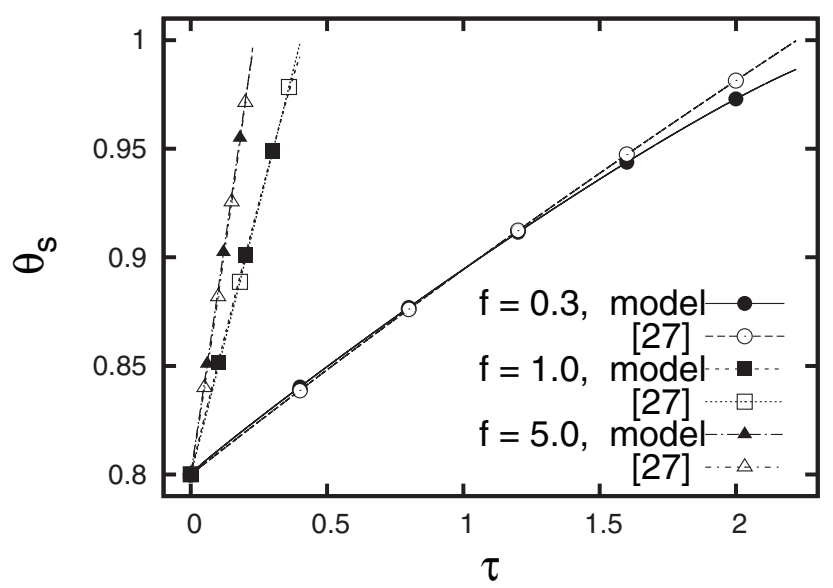

FIG. 7. Comparison of the results for the droplet surface temperature evolution from the present model and an analytical solution, ${ }^{27}$ for three different magnetic field frequencies $f=0.3,1.0,5.0$ and ambient temperature $\theta_{\infty}=1.0$. 


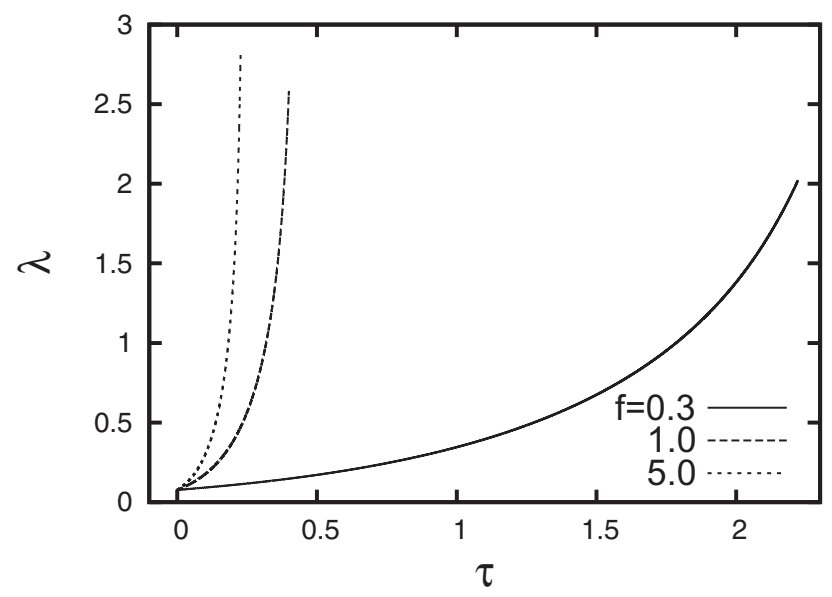

FIG. 8. Time evolution of the vaporization rate for three different magnetic field frequencies $f=0.3,1.0,5.0$ and ambient temperature $\theta_{\infty}=1.0$.

The value $q^{-}<0$ represents a heat loss from the droplet to the ambient atmosphere, whereas $q^{-}>0$ represents heat gain from gas phase. Figure 11 shows the heat flux across the droplet surface as a function of time for different conditions of the ambient temperature and the magnetic field frequency. For conditions where the ambient temperature is higher than the initial temperature of the droplet, the droplet initially receives heat from the ambient $\left(q^{-}>0\right)$. As the vaporization rate increases, vaporization heat loss leads to smaller values for the droplet surface temperature compared with that of the core $\left(q^{-}<0\right)$, as shown in Fig 11. Contrarily, in the case where the ambient temperature is lower than the initial temperature of the droplet, the droplet loses heat to the ambient during the whole heating period. The results reinforce the fact that the frequency $f$ controls the heating and vaporization of the droplet in low temperature atmosphere.

\section{Low ambient atmosphere temperature with $Y_{\infty}=0.2$}

Until now the vapor mass fraction in the ambient atmosphere was assumed to be zero. In this section, results for a ferrofluid droplet in an ambient atmosphere with temperature $\theta_{\infty}=0.75$ and fuel mass fraction $Y_{\infty}=0.2$ are presented. As gas phase is at a low temperature and the vapor

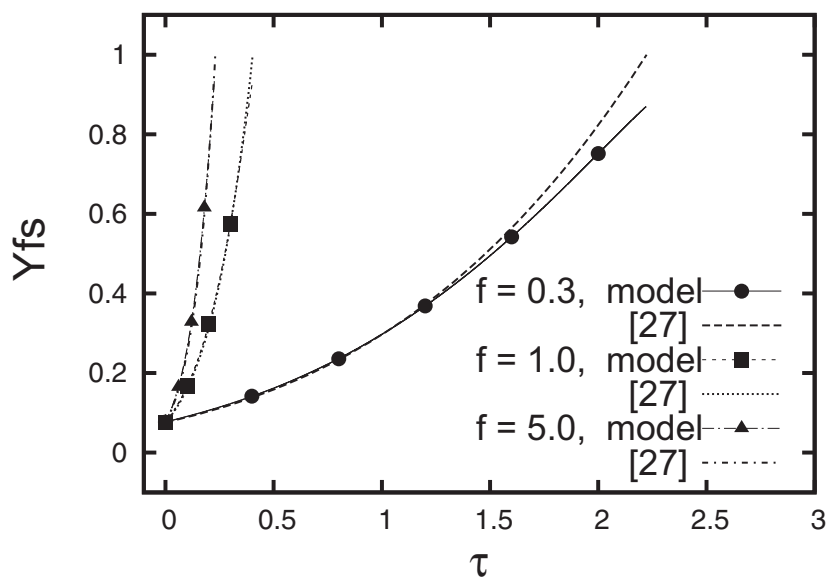

FIG. 9. Comparison of the results for the evolution of the fuel mass fraction at the droplet surface from the present model and an analytical solution, ${ }^{27}$ for three different magnetic field frequencies $f=0.3,1.0,5.0$, and ambient temperature $\theta_{\infty}=1.0$. 

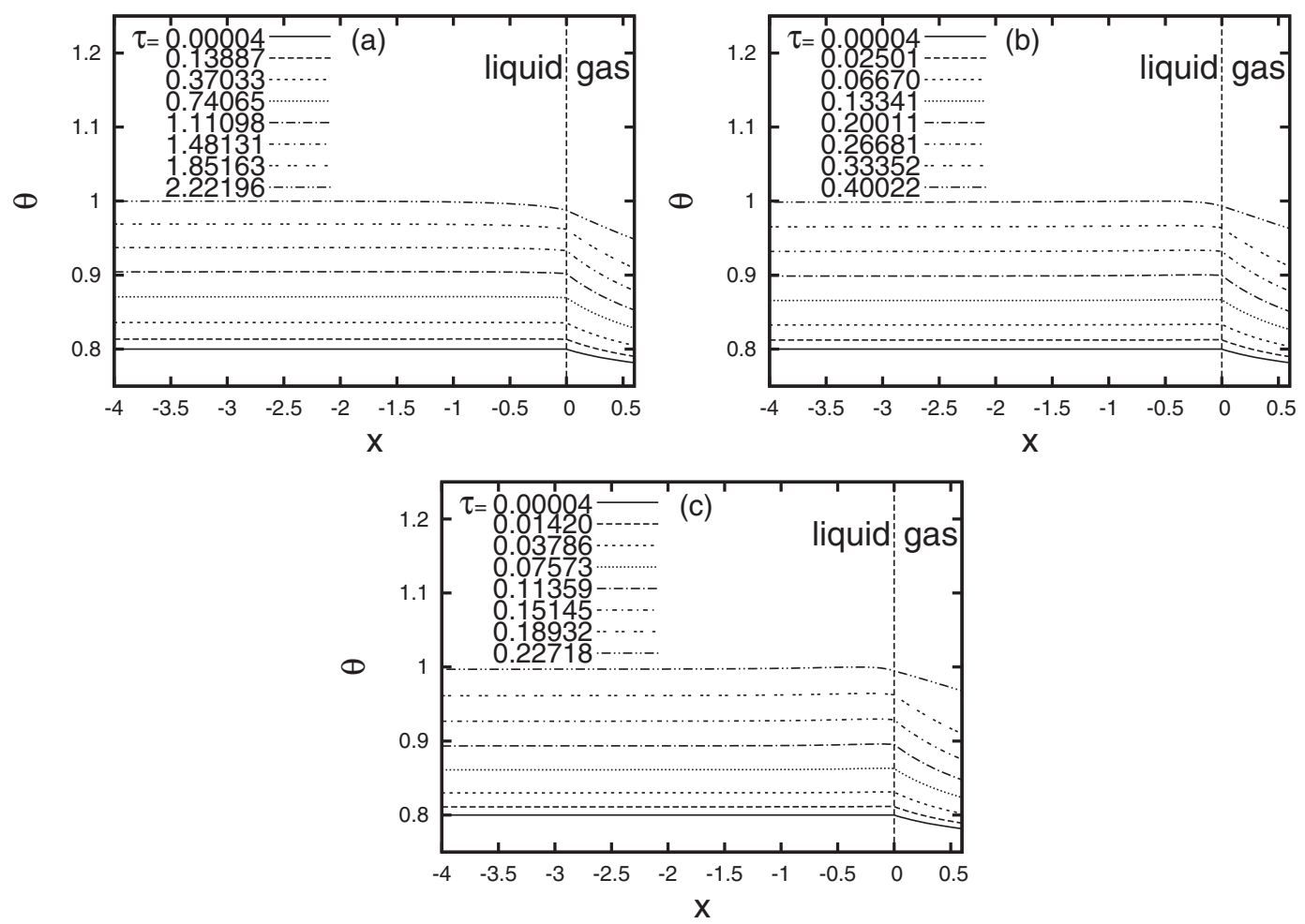

FIG. 10. Evolution of the temperature profile for three different magnetic field frequencies $f=0.3$ (a), 1.0 (b), and 5.0 (c), and $\theta_{\infty}=0.75$.

mass fraction at the droplet surface satisfies the condition $Y_{s}<0.2$, then the vapor near the droplet surface is condensed in the initial period of the heating process. In this work, the hypotheses that the condensed vapor on the droplet surface mixes instantaneously with the ferrofluid and the magnetic nanoparticles distribution does not change significantly are assumed. Without this consideration, the droplet would have a liquid layer on its surface with absence of nanoparticles. This feature is not covered by the present model.

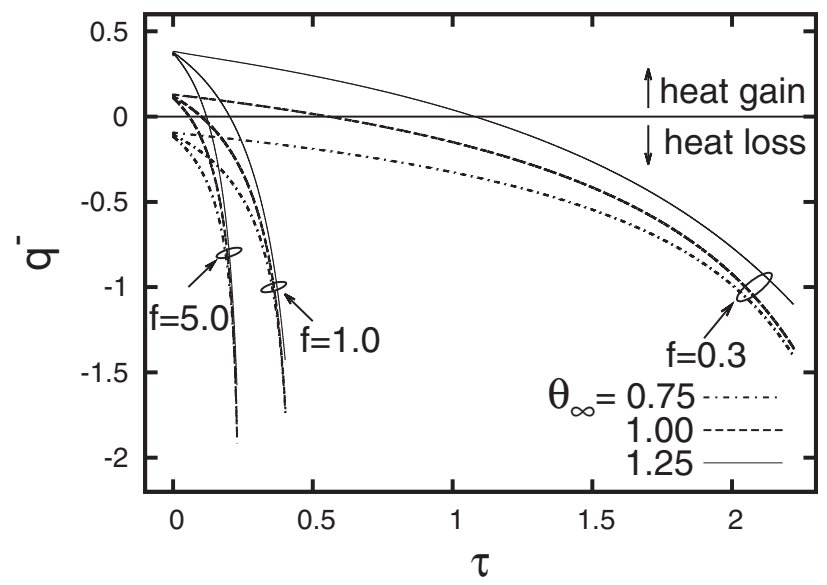

FIG. 11. Heat flux across the droplet surface as a function of time for different magnetic field frequencies $f=0.3,1.0,5.0$ under the conditions $\theta_{\infty}=0.75,1.0,1.25$. ( $q^{-}<0$ represents heat loss from the droplet to the ambient atmosphere and $q^{-}$ $>0$ represents heat gain from the gas phase.) 


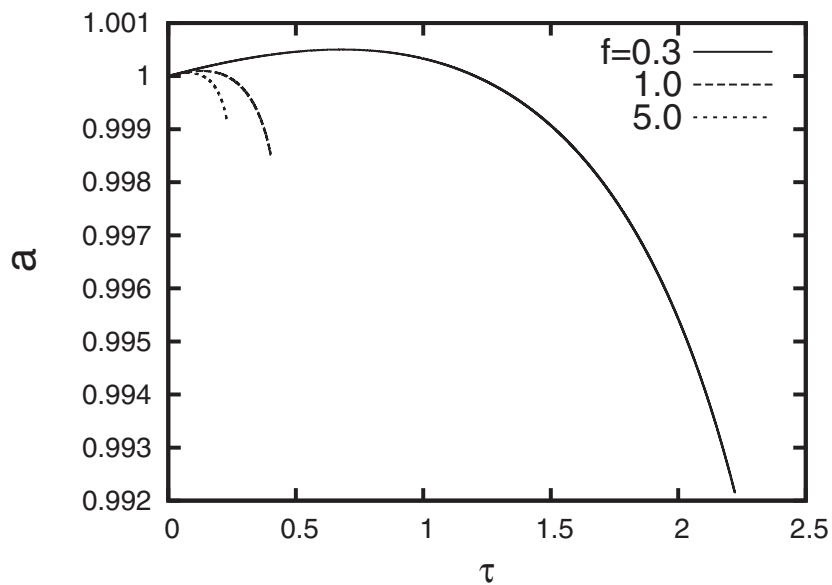

FIG. 12. Time evolution for the droplet radius for three different magnetic field frequencies $f=0.3,1.0,5.0$, ambient temperature $\theta_{\infty}=0.75$ and ambient fuel mass fraction $Y_{\infty}=0.2$.

It is known that, the surface of the droplet is heated during the condensation, but the temperature increment is insignificant compared with that imposed by the magnetic source. More precisely, for the condition $f=0.3$, the droplet surface presents an increase in temperature of the order of $10^{-5}$ in relation to the initial temperature inside of the droplet. Figure 12 shows the droplet radius as a function of time, from which an increase in the radius during condensation process is observed. Finally, Figure 13 shows the vaporization rate as a function of time. Negative values for the vaporization rate represents the condensation process. During the condensation, the mass flux of the vapor controls the droplet surface temperature and after that the magnetic source is responsible for increasing the droplet surface temperature. The behavior shown in Fig. 13 is similar to that presented in Fig. 8.

\section{E. Influence of the Lewis number}

The hypothesis of Lewis number equal to unity was considered to simulate the above cases, which is appropriate to some applications. The following results show the effect of Lewis number Le on temperature profiles in the thermal boundary layer. Figure 14 show the temperature profiles

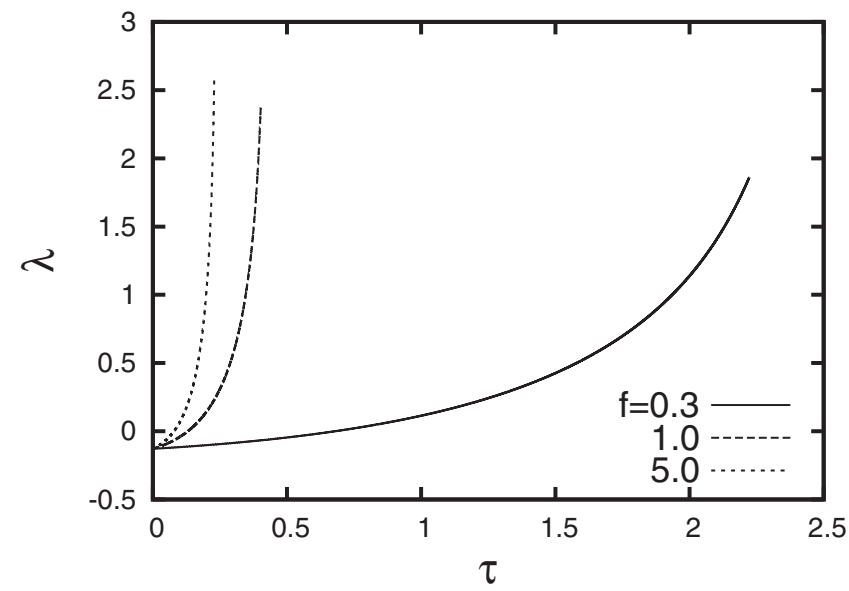

FIG. 13. Time evolution for the vaporization rate for three different magnetic field frequencies $f=0.3,1.0,5.0$, ambient temperature $\theta_{\infty}=0.75$ and ambient fuel mass fraction $Y_{\infty}=0.2$. 

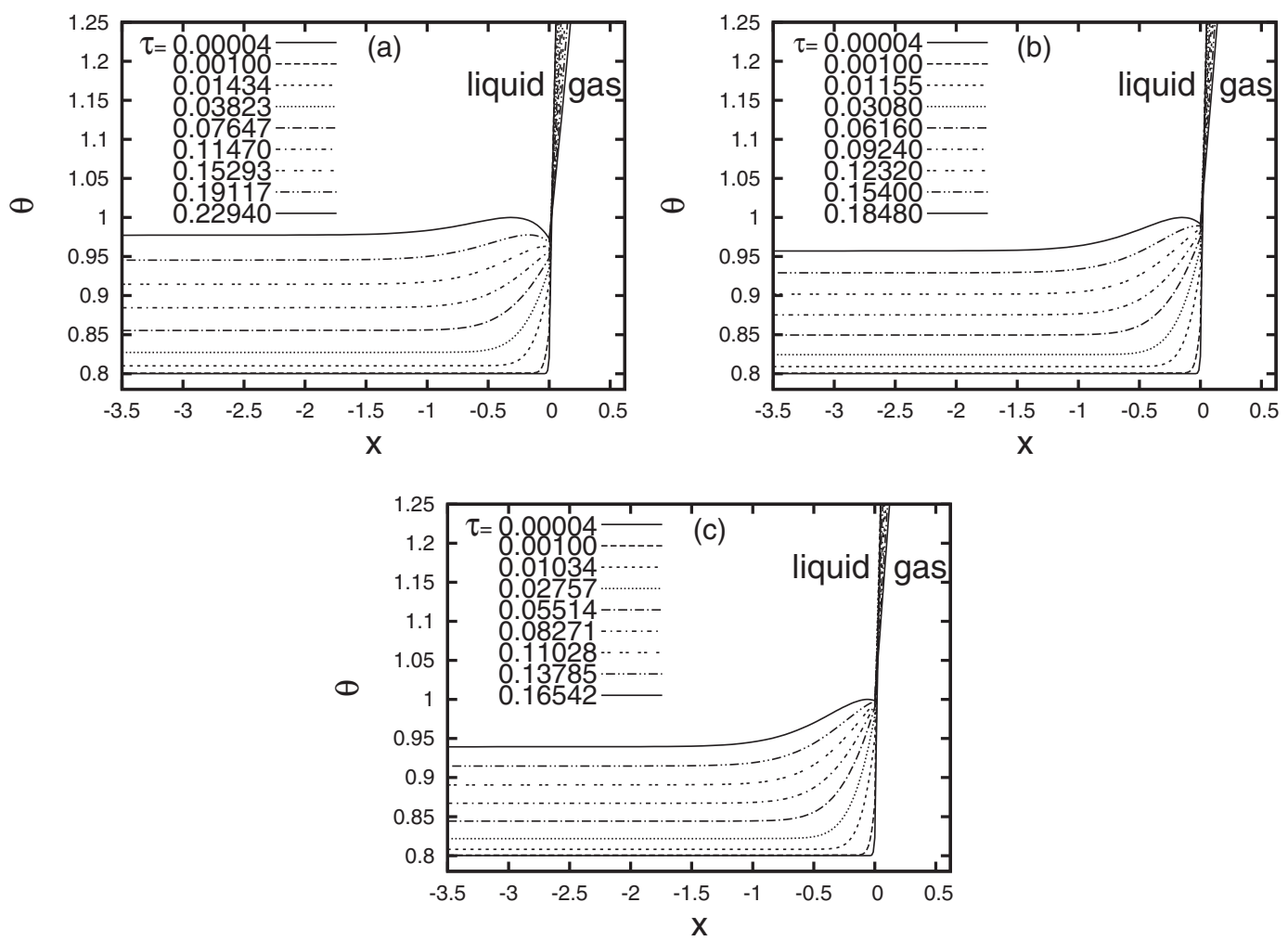

FIG. 14. Time evolution of the temperature profile for three different values of the Lewis number $L e=0.5$ (a), 1.0 (b), and 1.8 (c), magnetic field frequency $f=5.0$ and ambient temperature $\theta_{\infty}=6.0$.

during the ferrofluid droplet heating at high ambient temperature, $\theta=6.0$, and high magnetic field frequency, $f=5.0$, for $L e=0.5,1.0$, and 1.8. The results show that the Lewis number has a direct influence on the thermal boundary layer thickness and on the temperature profile. Since Lewis number measures the thermal diffusivity in relation to the mass diffusivity, then the vaporization rate, which depends on the gas phase mass diffusivity, increases as the Lewis number decreases. Consequently, due to the increase of the heat loss by the vaporization, the droplet surface temperature decreases. With the increasing temperature inside the thermal boundary layer and the decreasing droplet surface temperature, the heat transfer from the thermal boundary layer to the droplet surface increases by decreasing the vapor Lewis number, as seen in Fig. 14. This extra heat flux to the droplet surface augments the vaporization rate.

Figure 15 shows the thickness of the thermal boundary layer as a function of time for $L e=0.5$, 1.0, and 1.8. When the condition favors the augment of the droplet surface temperature $\theta_{s}(L e>1)$, the difference between $\theta_{s}$ and the droplet core temperature increases and, as a result, the thickness of the thermal boundary layer augments. In addition, since the heat loss by vaporization decreases the difference between the temperature surface and the droplet core temperature, the thickness thermal boundary layer decreases for $L e<1$.

Figures 16 and 17 show the vaporization rate and the droplet surface temperature, respectively, as functions of time for different values of Lewis number. Since the fuel mass diffusivity controls the droplet vaporization, the vaporization rate is larger for smaller values of the Lewis number, as shown in Fig. 16. However, the increase of the vaporization rate causes an increase on the droplet heat loss and a decrease on the droplet surface temperature, as seen in Fig. 17. Consequently, the intensity of the magnetic source is different inside the thermal boundary layer, leading to a local maximum on the temperature profile. 


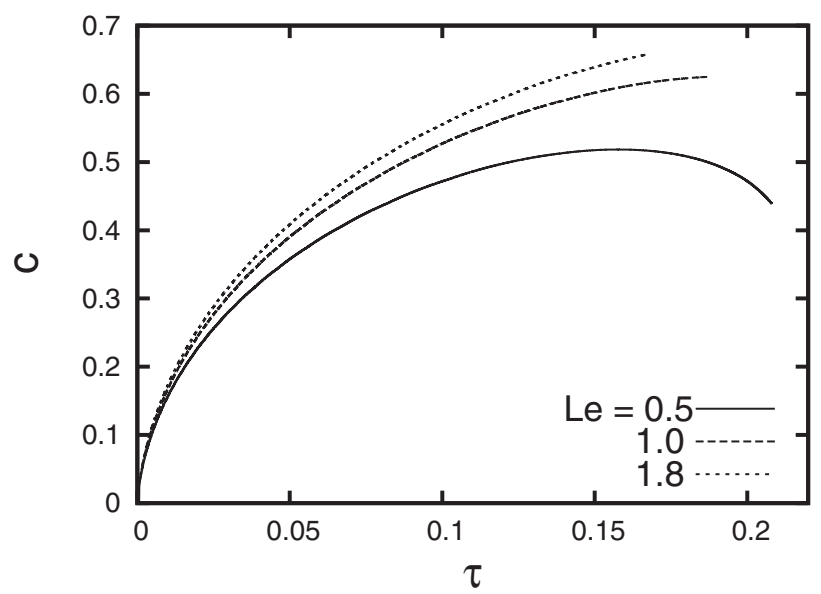

FIG. 15. Time evolution of the boundary layer thickness for three different Lewis number $L e=0.5,1.0$, and 1.8, magnetic field frequency $f=5.0$ and ambient temperature $\theta_{\infty}=6.0$.

\section{COMPARISON OF MAGNETIC AND LASER HEATING}

In this section, the magneto relaxation heating of ferrofluid droplet (water-maghtemite) is compared with the heating of pure water droplet irradiated by an unpolarizer plane wave $\mathrm{CO}_{2}$ laser beam. ${ }^{32-34}$ The case analyzed in laser heating considers a droplet of radius $a=10 \mu \mathrm{m}$, laser power of $10^{6} \mathrm{~W} / \mathrm{cm}^{2}$ and complete absorption of laser beam by the droplet. The droplet with initial temperature of $25^{\circ} \mathrm{C}$ is heated up to $305^{\circ} \mathrm{C}$ that is a value somewhat smaller than the superheat limit $T_{s u}=0.9 T_{c r}\left(T_{c r}\right.$ is the critical temperature and equal to $\left.373.85^{\circ} \mathrm{C}\right)$. These conditions are assigned for the case of the rapid heating of irradiated aerosols. ${ }^{34}$ Therefore, to compare magneto relaxation and laser heating, the former process will be permitted running up to the droplet core temperature to achieve $T=305^{\circ} \mathrm{C}$.

The comparison between the two heating methods is performed by comparing the results for $P_{m}$ and the dimensionless laser heat source $P_{L},{ }^{34}$ which is defined following the procedure adopted for the magnetic one:

$$
P_{L}=\frac{4 \pi \operatorname{Re}(n) \operatorname{Im}(n) S I / \lambda_{l w}}{\rho_{l} c_{l} T_{b} / t_{c}^{*}}
$$

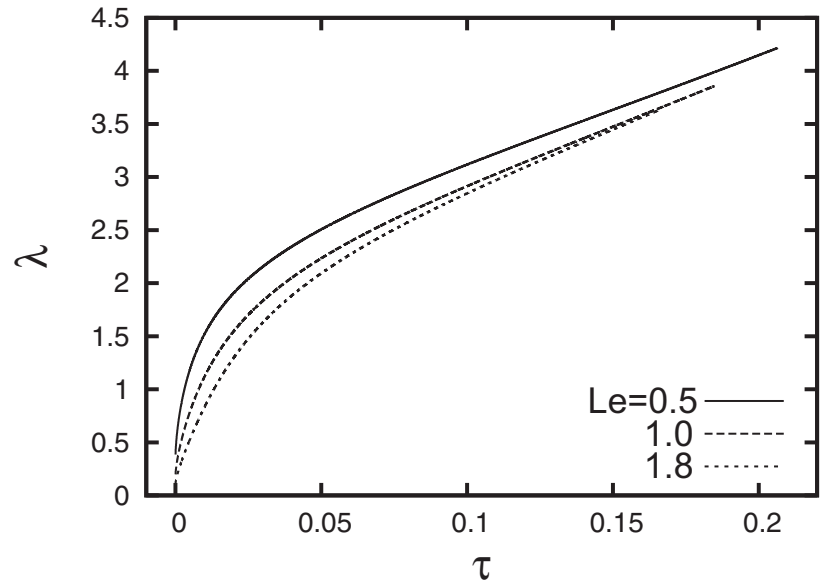

FIG. 16. Time evolution of the vaporization rate for three different Lewis number $L e=0.5,1.0$, and 1.8, magnetic field frequency $f=5.0$ and ambient temperature $\theta_{\infty}=6.0$. 


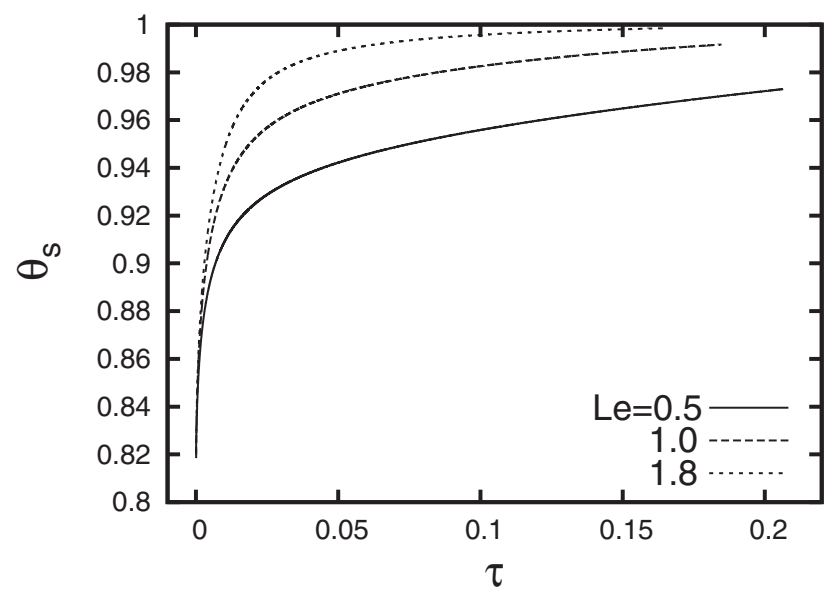

FIG. 17. Time evolution of the droplet surface temperature for three different Lewis number $L e=0.5,1.0$, and 1.8, magnetic field frequency $f=5.0$ and ambient temperature $\theta_{\infty}=6.0$.

in which $n=1.179-i 0.071, S=|E|^{2} /\left|E_{\text {inc }}\right|^{2}$ ( $E$ is the electric field at a point and $E_{\text {inc }}$ is the electric field of incident laser beam) and $\lambda_{l w}$ is the laser wavelength. The conditions addressed in the fast heating regime with $S=1$ leads to $P_{L}=3760$. The simulations point out a heating time about $1.41 \mu \mathrm{m} .{ }^{34}$ The simulations for magneto relaxation heating show the same heating time for $P_{m}=1950$ and magnetic field frequency $f=4$. This result indicates that magneto relaxation is able to heat up a larger droplet (about $a=300 \mu \mathrm{m}$ ) in the same time with $52 \%$ of source power. The comparison between the two simulations is exhibited in Fig. 18.

Not only difference on the parameter values is observed but also the behavior of the temperature profile close to the droplet surface. In the present model the term $\left(A P_{m}\right)^{1 / 2} \gg 1$, in the energy conservation condition at the droplet surface Eq. (9), demands a heat flux at the droplet surface in the liquid phase of order $\left(A P_{m}\right)^{-1 / 2} \ll 1$ to have order unity for that heat flux. The condition leads to a very small difference of temperature between the droplet surface and the droplet interior, explaining the small variation of temperature even close to the surface. In the case of laser heating, the temperature profile changes significantly close to the droplet surface and the reason for that

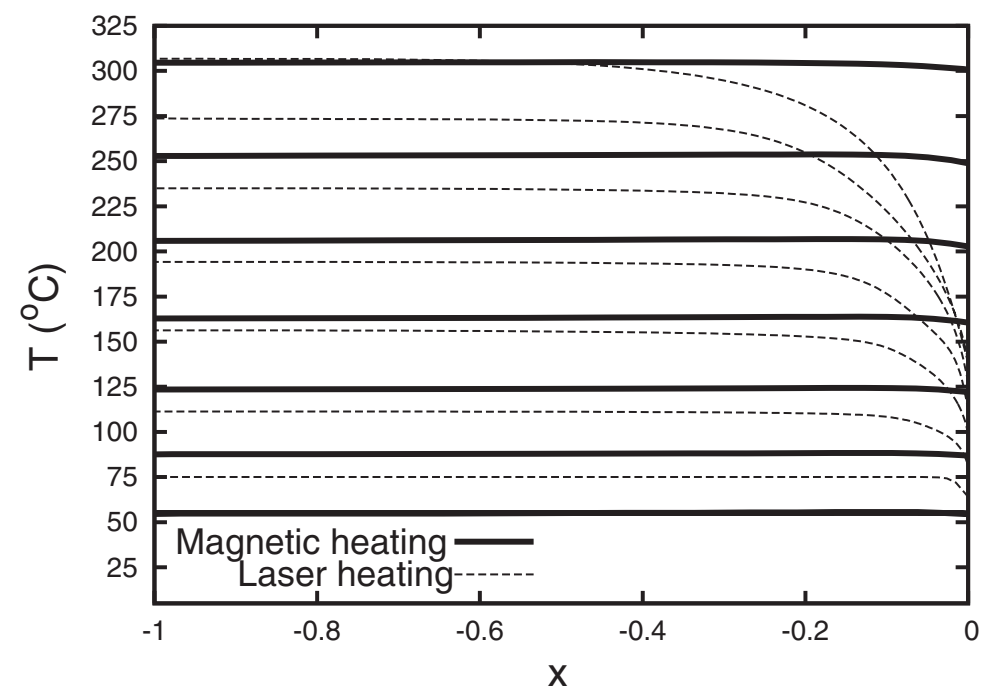

FIG. 18. Temperature profiles for magnetic relaxation heating $\left(P_{m}=1950\right.$ and $\left.f=4\right)$ and irradiated laser heating model ${ }^{34}$ at times, $t=0.2,0.4,0.6,0.8,1.0,1.2,1.4 \mu \mathrm{s}$. 
is different vaporization model adopted, which imposes a large vaporization rate resulting in low droplet surface temperature.

Since the laser source is considered constant in this case, the temperature variation with time is also constant. However, since the magnetic source depends on the temperature for high frequencies, the temperature evolution changes with time, as seen in Fig. 18.

\section{CONCLUSION}

Magneto relaxation heating on the droplet heating and vaporization processes is addressed in this work. It is observed that the magnetic energy source is responsible to heat uniformly the droplet interior. The heat flux from the ambient atmosphere is responsible to change the temperature profile only in a very thin layer close the surface. A thermal boundary layer is established. The combined heat sources inside the thermal boundary layer reduce the droplet heating time and increase the vaporization rate. The fact that the droplet interior reaches the boiling temperature rapidly can indicate formation of bubbles inside the droplet and its break into smaller droplets. Therefore, the magneto relaxation heating can be used in the future to improve also the atomization of fuels. In this work the results showed that the droplet surface temperature and the vaporization rate are strongly dependent on the Lewis number. For $L e<1$ an increase in the vaporization rate is obtained and consequently a decrease in the droplet surface temperature due to heat loss by vaporization. Increasing the Lewis number $L e>1$ the droplet surface temperature increases and the vaporization rate decreases. This favors that the droplet reaches the boiling temperature more quickly. By comparing the droplet heating produced by magneto relaxation and laser, it is seen advantages of magneto relaxation process because is possible to heat up a droplet at the same time but with less energy. Also, the advantage of magneto relaxation is to influence a large volume in space compared with the volume of the laser beam.

\section{ACKNOWLEDGMENTS}

Work supported partially by Conselho Nacional de Desenvolvimento Científico - CNPq under Grant Nos. 142110/2009-4, 563835/2008-7, and 303046/2010-4 and Coordenação de Aperfeiçoamento de Pessoal de Nível Superior - CAPES.

${ }^{1}$ R. Rosensweig, "Heating magnetic fluid with alternating magnetic field," J. Magn. Magn. Mater. 252, 370 (2002).

${ }^{2}$ D. Wen, G. Lin, S. Vafaei, and K. Zhang, "Review of nanofluids for heat transfer applications," Particuol. 7, 141 (2009).

${ }^{3}$ K. V. Wong and O. De Leon, "Applications of nanofluids: Current and future," Adv. Mech. Eng. 2010, 1.

${ }^{4}$ Y. Xuan, "Heat transfer enhancement of nanofluids," Int. J. Heat Fluid Flow 21, 58 (2000).

${ }^{5}$ Y. Xuan and W. Roetzel, "Conceptions for heat transfer correlation of nanofuids," Int. J. Heat Mass Transfer 43, 3701 (2000).

${ }^{6}$ Q. Z. Xue, "Model for effective thermal conductivity of nanofluids," Phys. Lett. A 307, 313 (2003).

${ }^{7} \mathrm{~B}$. Wang, "A fractal model for predicting the effective thermal conductivity of liquid with suspension of nanoparticles," Int. J. Heat Mass Transfer 46, 2665 (2003).

${ }^{8}$ Y. Feng, B. Yu, P. Xu, and M. Zou, "The effective thermal conductivity of nanofluids based on the nanolayer and the aggregation of nanoparticles," J. Phys. D: Appl. Phys. 40, 3164 (2007).

${ }^{9} \mathrm{~W}$. Yu, "The role of interfacial layers in the enhanced thermal conductivity of nanofluids: A renovated Maxwell model," J. Nanopart. Res. 5, 167 (2003).

${ }^{10}$ P. Tillman and J. Hill, "Determination of nanolayer thickness for a nanofluid," Int. Commun. Heat Mass Transfer 34, 399 (2007).

${ }^{11}$ Y. Zhang and H. B. Ma, "Nonequilibrium heat conduction in a nanofluid layer with periodic heat flux," Int. J. Heat Mass Transfer 51, 4862 (2008).

${ }^{12}$ E. Blums, S. Odenbach, A. Mezulis, and M. Maiorov, "Soret coefficient of nanoparticles in ferrofluids in the presence of a magnetic field," Phys. Fluids 10, 2155 (1998).

${ }^{13}$ M. Shliomis, "Ferrohydrodynamics: Retrospective and issues," Ferrofluids - Lect. Notes Phys. 594, 85 (2003).

${ }^{14} \mathrm{~T}$. Volker and S. Odenbach, "The influence of a uniform magnetic field on the Soret coefficient of magnetic nanoparticles," Phys. Fluids 15, 2198 (2003).

${ }^{15}$ L. Vekas, "Magnetic nanoparticles and concentrated magnetic nanofluids: Synthesis, properties and some applications," China Particuol. 5, 43 (2007).

${ }^{16}$ C. Ondeck, A. Habib, P. Ohodnicki, K. Miller, C. Sawyer, P. Chaudhary, and M. McHenry, "Theory of magnetic fluid heating with an alternating magnetic field with temperature dependent materials properties for self-regulated heating," J. Appl. Phys. 105, $07 \mathrm{~B} 324$ (2009). 
${ }^{17}$ J. A. Plaza, J. Esteve, P. Losantos, M. C. Acero, and C. Cane, "The use of ferrofluids in micromechanics," Sens. Actuators 84, $176(2000)$

${ }^{18}$ A. Hatch, A. Kamholz, G. Holman, P. Yager, and K. Bohringer, “A ferrofluidic magnetic micropump,” J. Microelectromech. Syst. 10, 215 (2001)

${ }^{19}$ C. Yamahata, M. Chastellain, V. Parashar, A. Petri, H. Hofmann, and M. Gijs, "Plastic micropump with ferrofluidic actuation," J. Microelectromech. Syst. 14, 96 (2005).

${ }^{20}$ B. U. Felderhof, "Ferrohydrodynamic pumping of a ferrofluid or electrohydrodynamic pumping of a polar liquid through a circular tube," Phys. Fluids 23, 092002 (2011).

${ }^{21}$ C. Y. Chen and C. S. Li, "Ordered microdroplet formations of thin ferrofluid layer breakups," Phys. Fluids 22, 014105 (2010).

${ }^{22}$ G. M. Faeth, "Current status of droplet and liquid combustion,” Prog. Energy Combust. Sci. 3, 191 (1977).

${ }^{23}$ W. A. Sirignano, "Fuel droplet vaporization and spray combustion theory," Prog. Energy Combust. Sci. 9, 291 (1983).

${ }^{24}$ S. S. Sazhin, "Advanced models of fuel droplet heating and evaporation," Prog. Energy Combust. Sci 32, 162 (2006).

${ }^{25}$ S. Maenosono and S. Saita, "Theoretical assessment of FePt nanoparticles as heating elements for magnetic hyperthermia," IEEE Trans. Magn. 42, 1638 (2006).

${ }^{26}$ R. Kappiyoor, M. Liangruksa, R. Ganguly, and I. K. Puri, "The effects of magnetic nanoparticle properties on magnetic fluid hyperthermia," J. Appl. Phys. 108, 094702 (2010).

${ }^{27}$ F. F. Fachini and A. F. Bakuzis, "Decreasing nanofluid droplet heating time with alternating magnetic fields," J. Appl. Phys. 108, 084309 (2010).

${ }^{28}$ Y. Gan and L. Qiao, "Combustion characteristics of fuel droplets with addition of nano and micron-sized aluminum particles," Combust. Flame 158, 354 (2011).

${ }^{29}$ F. F. Fachini, "An analytical solution for the quasi-steady droplet combustion," Combust. Flame 116, 302 (1999)

${ }^{30}$ F. F. Fachini, A. Liñán, and F. A. Williams, "Theory of flame histories in droplet combustion at small stoichiometric fuel-air ratios," AIAA J. 37, 1426 (1999).

${ }^{31}$ C. C. Tseng and R. Viskanta, "Enhancement of water droplet evaporation by radiation absorption," Fire Saf. J. 41, 236 (2006).

${ }^{32}$ R. L. Armstrong, "Interactions of absorbing aerosols with intense light beams," Appl. Opt. 56, 2142 (1984).

${ }^{33}$ R. L. Armstrong, P. J. O'Rourke, and A. Zardecki, "Vaporization of irradiated droplets," Phys. Fluids 29, 3573 (1986).

${ }^{34}$ B. S. Park and R. L. Armstrong, "Laser droplet heating: fast and slow heating regimes," Appl. Opt. 28, 3671 (1989). 